\title{
Gestion post-récolte des graines d'Acacia macrostachya Reichenb. ex DC. dans la province du Boulkiemdé au Burkina Faso : diagnostic participatif en milieu paysan
}

\author{
Marcellin YAMKOULGA (1,2), Antoine WAONGO (3), Louis SAWADOGO (1) et Antoine SANON (2) \\ (1) Centre National de la Recherche Scientifique et Technologique (CNRST), Institut de l'Environnement et de Recherches \\ Agricoles (INERA), Département Environnement et Forêt (DEF), 03 BP 7047 Ouagadougou 03, Burkina Faso \\ (2) Laboratoire d'Entomologie Fondamentale et Appliquée (LEFA), UFR/SVT Université Ouaga1 Pr Joseph KI-ZERBO, 06 \\ BP 9499 Ouagadougou 06, Burkina Faso \\ (3) Laboratoire Central d'Entomologie Agricole de Kamboinsé (LCEAK), Institut de l'Environnement et de Recherches \\ Agricoles (INERA), 04 BP 8645 Ouagadougou 04, Burkina Faso \\ Auteur correspondant e-mail : marcellin_yamkoulga@yahoo.fr Tél : +226 70327572
}

Original submitted in on $17^{\text {th }}$ June 2018. Published online at www.m.elewa.org on $31^{\text {st }}$ October 2018 https://dx.doi.org/10.4314/jab.v130i1.3

\section{RÉSUMÉ}

Objectifs: Les objectifs de cette étude étaient d'inventorier les différentes pratiques post-récoltes d'A. macrostachya; les contraintes liées au stockage ; les méthodes de protection des stocks et les différents usages des graines.

Méthodologie et résultats : une enquête a été réalisée dans vingt villages de la province du Boulkiemdé auprès des acteurs de la gestion post récolte des graines d'A. macrostachya. Les résultats ont montré que $52,13 \%$ des répondants conservent leurs graines dans les sacs en polypropylène, $24,47 \%$ dans les bidons de 201 de contenance et $20,21 \%$ dans les jarres. Les insectes sont perçus comme les principaux ravageurs des graines. Leurs dégâts commencent avant la récolte des gousses et se poursuivent dans les stocks tout au long du stockage. La méthode de protection la plus utilisée pour faire face aux ravageurs est la précuisson à la chaleur. En l'absence de méthode de lutte, des dégâts importants sont observables dès la première semaine de stockage. La conservation des graines répond à l'objectif de satisfaire les besoins de consommation et de commercialisation.

Conclusion et application : Cette étude a permis de mieux appréhender les pratiques paysannes en gestion post récolte et les contraintes de stockage des graines de $A$. macrostachya. Elle ouvre les perspectives d'une gestion plus efficiente de cette importante denrée alimentaire.

Mots clés : Graines de zamné, gestion post-récolte, méthodes de conservation, insectes ravageurs, perception paysanne, Burkina Faso. 
Yamkoulga et al., J. Appl. Biosci. 2018 Gestion post-récolte des graines d'Acacia macrostachya Reichenb. ex DC. dans la province du Boulkiemdé au Burkina Faso : diagnostic participatif en milieu paysan

Post-harvest management of the seeds of Acacia macrostachya Reichenb. ex DC. in the Boulkiemdé province in Burkina Faso : participatory survey among farmers

\begin{abstract}
Objectives: The objectives of this survey were to inventory the different post-harvest practices of $A$. macrostachya; the storage problems; control methods use to protect $A$. macrostachya during storage and the different uses of seeds

Method and results : A survey was conducted in 20 villages in the province of Boulkiemdé among the actors of A. macrostachya seeds post-harvest management. The results showed that $52.13 \%$ of respondents kept their seeds in polypropylene bags, $24.47 \%$ in cans of 201 capacity and $20.21 \%$ in jars. Insects are considered as the main pests of $A$. macrostachya seeds. Damage begins before the harvest of the pods and continues during post-harvest storage. The most widespread method of protection for pests is pre-cooking with heat. In the absence of a control method, a lot damage can be observed from the first week of storage. Seed conservation serves the purpose of meeting consumer and marketing needs.

Conclusion and application : This study made it possible to better understand farmers' practices in the postharvest management and the storage problems of $A$. macrostachya seeds. It opens up prospects for more efficient management of this important food.
\end{abstract}

Keywords: zamné seeds, post-harvest management, conservation methods, insect pests, farmer perception, Burkina Faso.

\section{INTRODUCTION}

Le monde d'aujourd'hui est confronté à deux défis de taille à savoir assurer la sécurité alimentaire et une alimentation équilibrée à tous. En effet, selon I'Organisation des Nations Unies pour l'alimentation et l'agriculture (FAO, 2016), 800 millions de personnes souffrent de faim chronique et environ deux milliards de personnes souffrent d'au moins une carence en micronutriments. II faut donc de toute urgence une intervention concertée pour affronter la question de la sécurité alimentaire et nutritionnelle au niveau mondial (Hunter et Fanzo, 2013). Dans cette optique, une attention particulière doit être portée aux ligneux alimentaires car ils sont sources d'alternative et de variation de régimes alimentaires et aussi de revenus et d'emplois pour les populations locales (Thiombiano et al., 2012). Au Burkina Faso, parmi ces ligneux alimentaires, Acacia macrostachya joue un rôle capital en raison de ses nombreuses potentialités. C'est une légumineuse alimentaire répartie à peu près uniformément dans l'ensemble du pays (Arbonnier, 2000 ; Wittig et al., 2004). L'intérêt porté à ce végétal tient en ce que ses produits forestiers non ligneux (PFNL) notamment ses graines jouent plusieurs rôles. Riches en protéines, fer, calcium, potassium, magnésium, phosphore mais également en vitamines A et $E$ (Savadogo et al., 2011), ses graines sont consommées de plus en plus sous forme de ragout ou de plats associés à des céréales au cours des grandes cérémonies coutumières et religieuses indiquant leur attachement culturel (Hama-Ba et al., 2017). Sur le plan économique, la commercialisation des graines génère des revenus substantiels pour la population rurale (Hagberg et al., 1996 ; Kristensen et Balslev, 2003). Plusieurs études ont été entreprises pour améliorer la productivité et assurer la régénérescence de cette légumineuse (Zida, 2007; Schelin et al, 2004). Malgré les initiatives prises de part et d'autre pour accroitre la production, le contexte d'insécurité alimentaire est toujours marqué par des pertes post-récolte non négligeables (Gueye et al., 2011). Les pertes enregistrées sont de plus en plus croissantes, car les technologies traditionnelles de stockage et de transformation mises en œuvre sont généralement inadéquates avec des risques d'infestation des produits stockés (Fandohan et al., 2003). Ces pertes interviennent en effet, à tous les stades, de la récolte à la consommation : d'abord chez le producteur, qu'il s'agisse de produits destinés à l'autoconsommation, à la semence ou en attente d'être commercialisés ; puis pendant le transport vers les lieux de stockage 
Yamkoulga et al., J. Appl. Biosci. 2018 Gestion post-récolte des graines d'Acacia macrostachya Reichenb. ex DC. dans la province du Boulkiemdé au Burkina Faso : diagnostic participatif en milieu paysan

et au cours du stockage ; enfin dans les réserves des commerçants (Sembène, 1996). Les principaux insectes ravageurs des stocks de légumineuses appartiennent à la famille des Bruchidae (El-Miziani et al., 2016). Les dégâts occasionnés à ces légumineuses entreposées par les bruches sont très importants. Ainsi, l'infestation des graines d'A. macrostachya par les larves de bruches réduit significativement le taux de germination des graines (Schelin et al., 2004) ce qui impacte négativement sur sa régénérescence. En dépit de l'effet néfaste des ravageurs, les graines d'A. macrostachya sont quand même consommées au Burkina Faso durant toute l'année sous diverses formes, ce qui suppose l'existence de stratégies de lutte endogènes utilisées par la population rurale pour faire face à ces insectes. Ces stratégies de lutte de même que les différentes pratiques post-récoltes sont malheureusement mal connues et peu documentées. Or certaines des méthodes de lutte pourraient présenter des risques pour la santé des consommateurs. Connaître les pratiques postrécoltes devient alors un impératif pour valoriser la denrée tant sur le plan alimentaire qu'économique. L'objectif général de cette étude était de contribuer à mieux connaître la problématique de la gestion postrécolte des graines d'A. macrostachya dans la province du Boulkiemdé au Burkina Faso à travers une enquête en milieu rural. De façon spécifique, nous avons inventorié $(i)$ les différentes pratiques post-récoltes d'A. macrostachya ; (ii) les contraintes liées au stockage ; (iii) les méthodes de protection des stocks et (iv) les différents usages et la valeur marchande des graines.
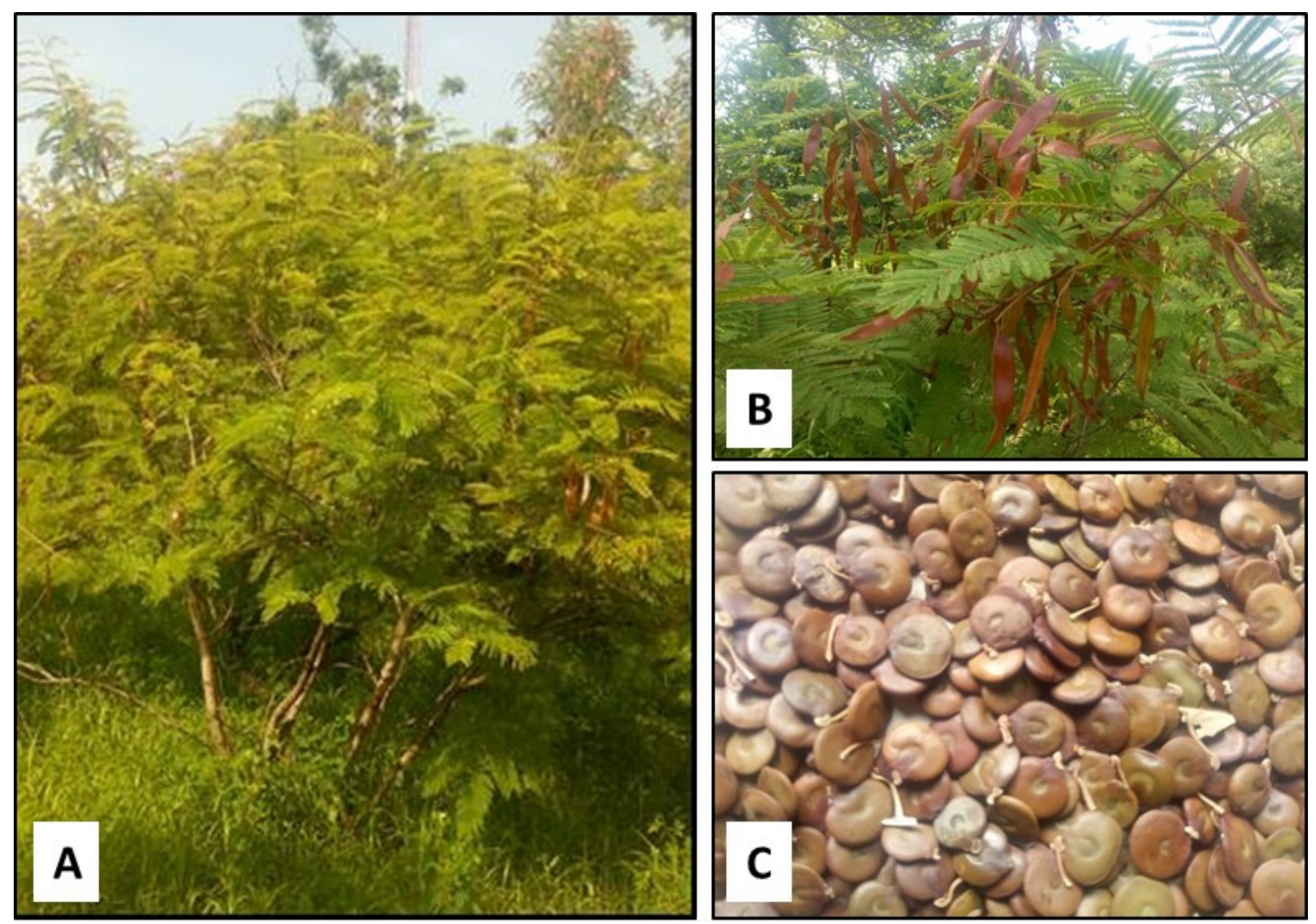

Photo 1 : quelques pieds $(A)$, gousses $(B)$ et graines saines $(C)$ d'A. macrostachya 


\section{MATÉRIEL ET MÉTHODES}

Description de la zone d'étude : L'étude a été conduite dans la région du centre ouest du Burkina Faso précisément dans la province du Boulkiemdé. Celle-ci est située dans le domaine phytogéographique de type nord soudanien avec une pluviométrie moyenne annuelle variant entre 700 et $900 \mathrm{~mm}$ (Fontès et Guinko, 1995) et une saison sèche de 6 à 7 mois. Selon l'Institut National de la Statistique et de la Démographie (INSD, 2016), le Boulkiemdé comptait en 2015,630 922 habitants répartis dans 15 communes. Vingt villages répartis dans sept (07) communes sont concernées par l'enquête à savoir la zone rurale de Koudougou, les communes rurales de Soaw, Kindi, Pella, Nanoro, Poa et Nandiala (Figure 1).

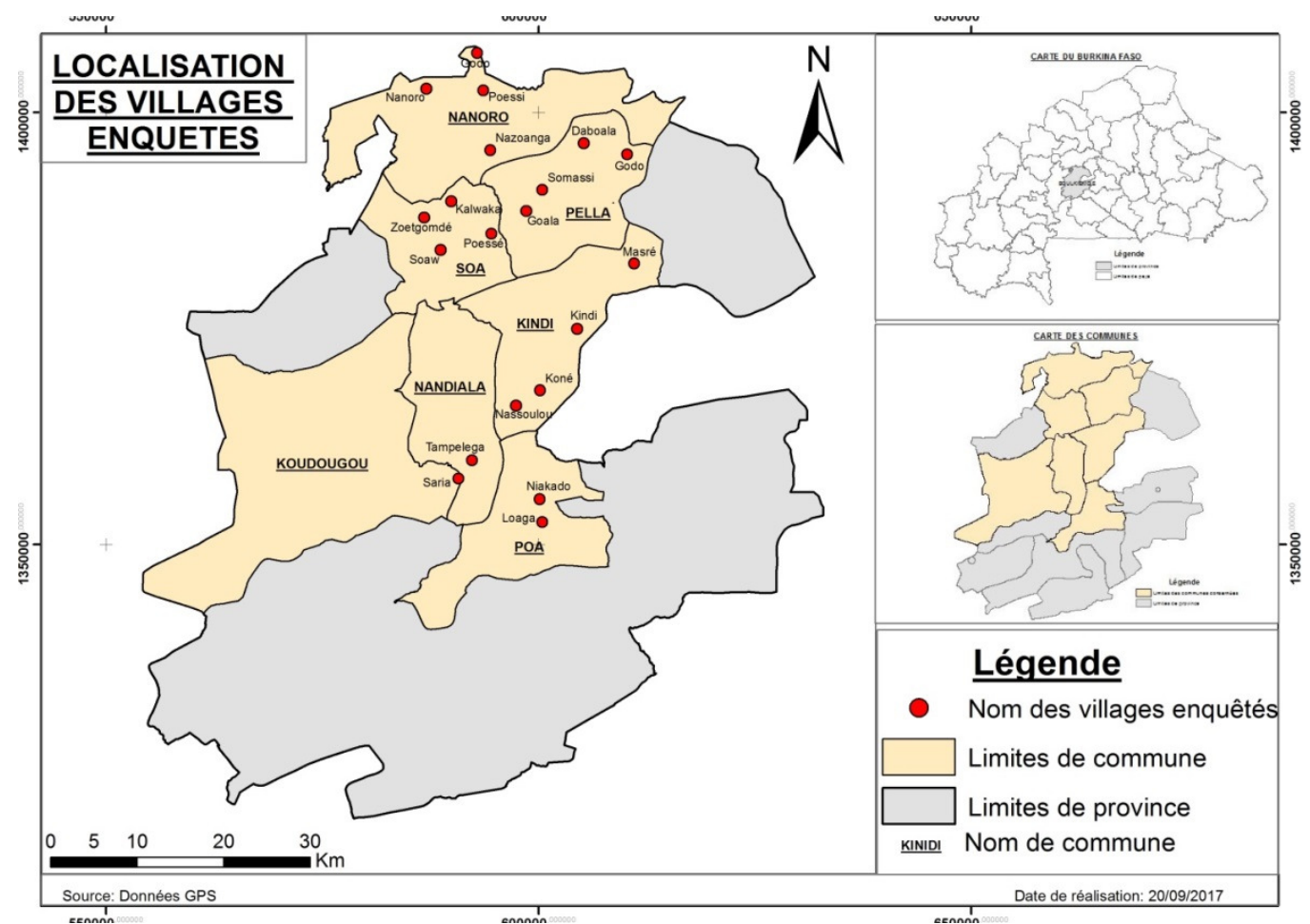

Figure 1 : carte de la province du Boulkiemdé mettant en évidence les villages enquêtés

Détermination de la taille de l'échantillonnage : La détermination de la taille de l'échantillon a été réalisée selon la méthode décrite par Dagnelie (1998). Elle a consisté à réaliser une enquête préliminaire dans cinq villages choisis de façon aléatoire auprès de cent (100) personnes soit vingt (20) personnes dans chaque village. Elle a permis de déterminer la proportion des individus chez qui les insectes constituent ou non, un frein à la conservation des graines d'A. macrostachya. Les $97 \%$ de personnes qui ont répondu par l'affirmative, ont été utilisés pour déterminer la taille de l'échantillon en exploitant la formule suivante (Dagnelie, 1998) :

$N=\frac{\mu_{1-\alpha / 2}^{2} P i(1-P i)}{d^{2}}$

Où $N=$ nombre total de personnes à enquêter représentant la taille de l'échantillon ; $\mu_{1-a / 2}$ représente la valeur de la variable aléatoire normale pour un risque a égal à $0,05 .\left(\mu_{1-\alpha / 2}=1,96\right) ; d=$ marge d'erreur considérée dans l'enquête, $1 \%<d<15 \%$. On prendra $d$ $=0,03 ; P i=$ proportion des personnes qui ont affirmé que sans méthode(s) de lutte, les stocks d'A. macrostachya sont fortement ravagés par les insectes. La taille de l'échantillon à enquêter en utilisant la formule de Dagnelie (1998) est de 124 personnes. Le nombre de personnes à enquêter par commune a été déterminé par proportionnalité en tenant compte de la taille de la population de chaque localité (INSD, 2006). Ainsi, le nombre de personnes à enquêter était de 30 dans la zone rurale de Koudougou, 10 à Soaw, 12 à Pella, 20 à Kindi,20 à Nanoro, 14 à Nandiala et 18 à Poa.

Diagnostic de la gestion post-récolte des graines d'A. macrostachya en milieu paysan : Les vingt villages à enquêter ont été choisis de concert avec les agents 
d'agriculture, en raison de la présence de peuplements importants de l'espèce et de la disponibilité des graines. Un questionnaire a été élaboré et les principales informations recueillies étaient relatives aux caractéristiques générales des enquêtés, aux différentes pratiques des producteurs en lien avec la denrée depuis la récolte jusqu'au stockage, les structures de stockage et les méthodes de lutte ainsi que la destinée des stocks. Le questionnaire a été administré à chaque enquêté de façon individuelle pour éviter une influence des uns sur les autres.

Analyse statistique des données : Une vérification de la distribution des données avec le test de Shapiro - Wilk

\section{RÉSULTATS}

Caractéristiques générales des enquêtés : II ressort de l'analyse du tableau 1 que le sexe féminin représente la majorité de la population enquêtée $(87,10 \%)$. Dans cette population, la tranche d'âge comprise entre 40 et 60 ans est la plus représentée $(66,13 \%)$. En plus d'être en en utilisant la fonction Shapiro. test a été réalisé sur le prix d'une assiettée yoruba et la durée de conservation des graines d'A. macrostachya afin de choisir le test approprié. Ainsi, Le test de Mann - Whitney - Wilcoxon a été réalisé avec la fonction wilcox.test au seuil de probabilité de $5 \%$. Les traitements statistiques ont été réalisés avec le logiciel $R$ version 3.4 .3 (2017-11-30). Toutes les autres données collectées lors de l'enquête ont été saisies dans le tableur Excel 2013 qui a par la suite servi à calculer les différentes proportions des réponses aux questions posées et de construire les différents graphiques.

majorité analphabète ( $83,87 \%)$, les individus enquêtés ne sont pas organisés en coopérative. On rencontre cependant quelques individus qui ont le niveau primaire et quelques rares alphabétisés ou ayant le niveau secondaire.

Tableau 1 : Caractéristiques de la population enquêtée

\begin{tabular}{|l|c|c|}
\hline Variables & Fréquence & Pourcentage (\%) \\
\hline Sexe & & 12,9 \\
\hline Masculin & 16 & 87,10 \\
\hline Féminin & 108 & \\
\hline Âge & & 29,03 \\
\hline$[20-40[$ & 36 & 66,13 \\
\hline$[40-60[$ & 82 & 4,84 \\
\hline$\geq 60$ & 6 & \\
\hline Niveau d'instruction & & 83,87 \\
\hline Néant & 104 & 3,23 \\
\hline Alphabétisé & 4 & 8,87 \\
\hline Primaire & 11 & 4,03 \\
\hline Secondaire & 5 & \\
\hline Organisation en coopérative & 13 & 10,48 \\
\hline Oui & 111 & 89,52 \\
\hline Non & & \\
\hline
\end{tabular}

Analyse des pratiques post-récolte: L'analyse des différentes pratiques post-récolte avant la conservation (Tableau 2) montre que la majorité des producteurs sèchent les gousses, les battent et les vannent, puis effectuent un séchage complémentaire des graines avant la conservation $(57,99 \%)$. Deux autres pratiques sont aussi très représentées. II s'agit notamment (1) du battage et du vannage des gousses immédiatement après la récolte suivi du séchage complémentaire des graines, et (2) du séchage des gousses suivi du battage et du vannage avec respectivement $23,67 \%$ et $16,57 \%$ des enquêtés. II est à noter qu'une minorité de producteurs stockent les gousses $(1,18 \%)$. Le séchage de ces graines qui s'effectue préférentiellement à la maison (Tableau 2), dure en moyenne entre 1 et 7 jours pour $80,64 \%$ des enquêtés. En revanche, $16,13 \%$ et $3,23 \%$ des enquêtés affirment que la durée du séchage varie respectivement entre 7-14 jours et 14-21 jours (Figure 2). 


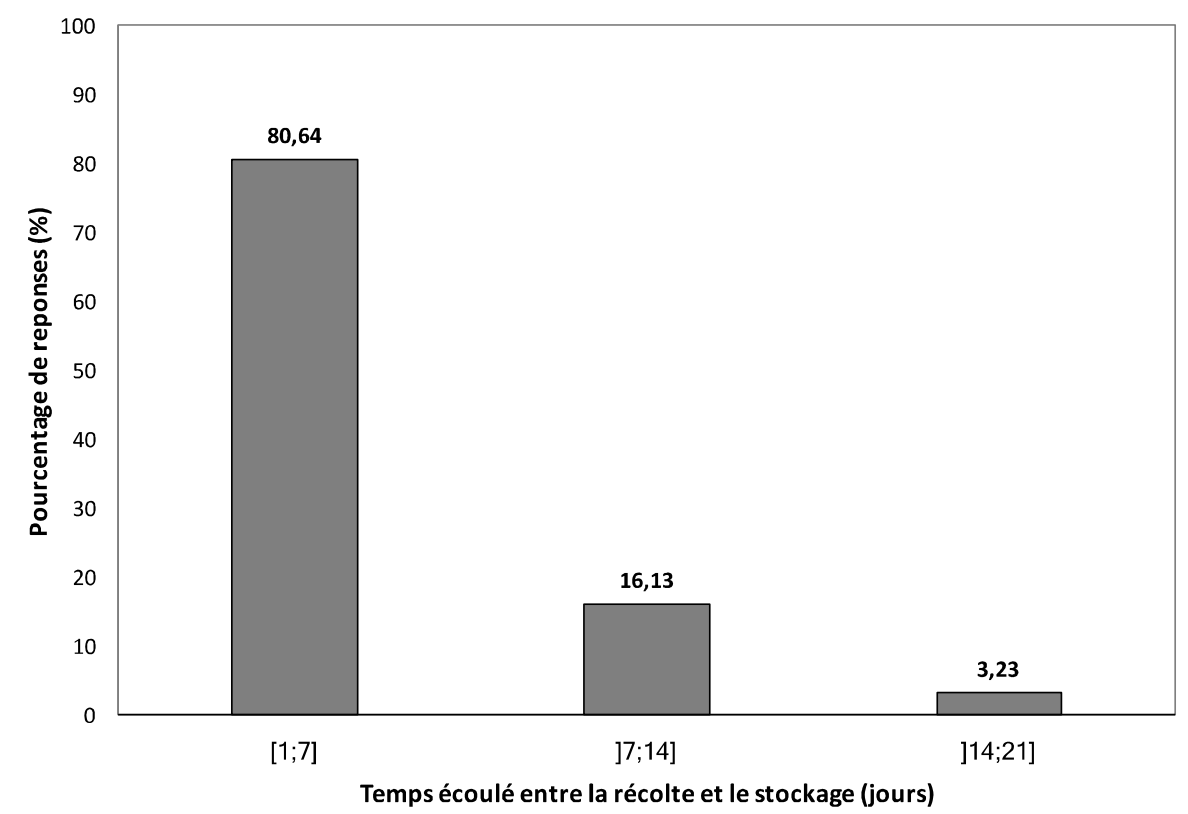

Figure 2 : Répartition des réponses des enquêtés sur la durée de séchage des gousses avant leur conservation

Tableau 2 : Répartition des réponses des enquêtés entre les pratiques post-récoltes réalisées avant la conservation des graines

\begin{tabular}{|l|c|}
\hline \multicolumn{1}{|c|}{ Opérations post-récoltes } & Proportion des réponses (\%) \\
\hline \multicolumn{1}{|c|}{ Pratiques post-récoltes } & 57,99 \\
\hline $\begin{array}{l}\text { Séchage des gousses, battage et vannage puis séchage } \\
\text { complémentaire des graines }\end{array}$ & 23,67 \\
\hline $\begin{array}{l}\text { Battage des gousses immédiatement après la récolte puis } \\
\text { séchage des graines après vannage }\end{array}$ & 16,57 \\
\hline $\begin{array}{l}\text { Séchage des gousses puis battage, vannage et } \\
\text { conservation des graines }\end{array}$ & 1,18 \\
\hline Séchage et conservation des gousses & 0,59 \\
\hline $\begin{array}{l}\text { Battage des gousses immédiatement après la récolte puis } \\
\text { vannage et conservation des graines }\end{array}$ & \\
\hline \multicolumn{1}{|c|}{ Lieu de séchage des gousses } \\
\hline Maison & 89,13 \\
\hline Champ & 10,87 \\
\hline
\end{tabular}

Structures et modes de stockage des graines d'Acacia macrostachya

Structures de stockage: L'examen de la figure 3 montre qu'il existe principalement quatre types de structures de stockage qui n'ont cependant pas les mêmes proportions d'utilisation. Les structures les plus utilisées sont les sacs en polypropylène $(52,13 \%$ des enquêtés). Viennent ensuite les bidons en plastique de 20 litres de contenance, puis les jarres en terre cuite et enfin les greniers avec respectivement des proportions de $24,47 \% ; 20,21 \%$ et $3,19 \%$. 


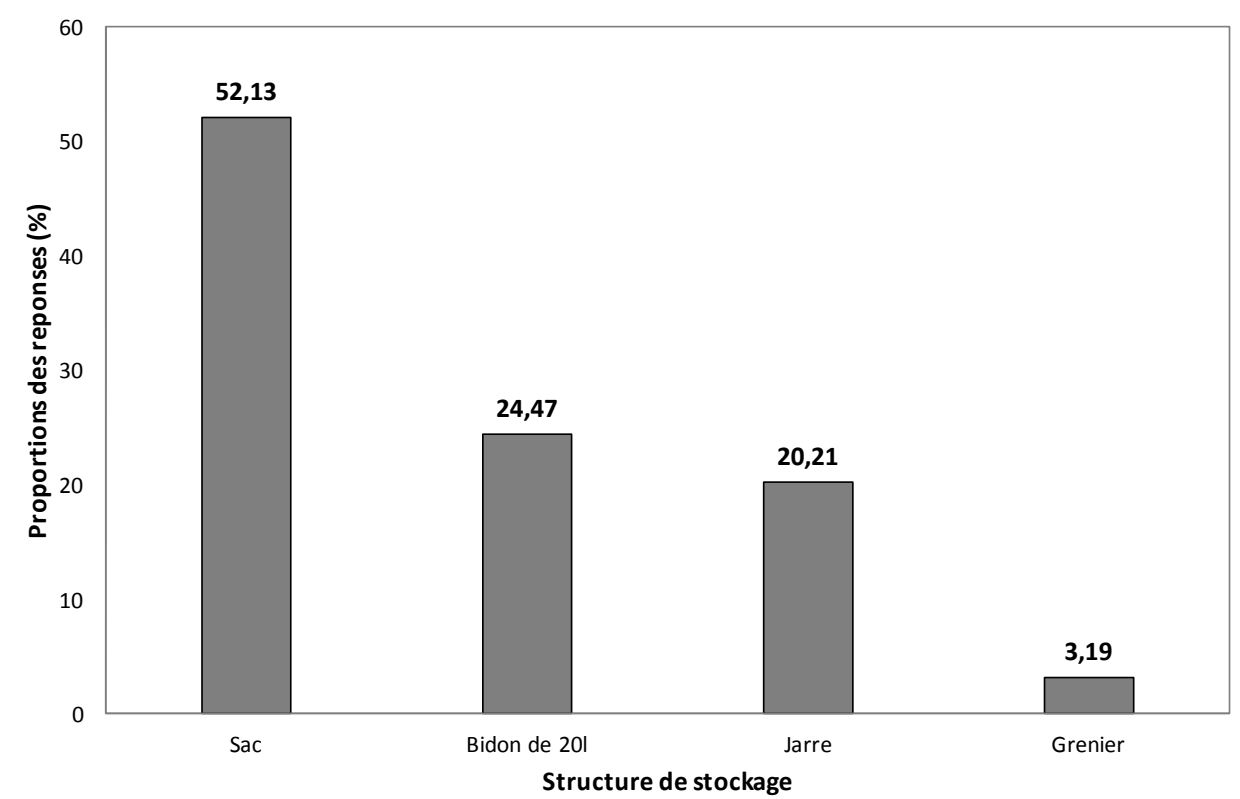

Figure 3 : Répartition des réponses des enquêtés sur les structures de stockage des graines d'A. macrostachya

Pratiques liées au stockage : Les résultats consignés dans le tableau 3 montrent comment les exploitants stockent les graines et/ou les gousses d'A. macrostachya. La majorité des exploitants ( $81,62 \%$ des réponses) n'effectuent pas de mélange avec d'autres denrées pour la conservation. Cependant la minorité qui effectue un mélange le fait surtout avec le niébé ( $36 \%$ des réponses) et l'arachide ( $28 \%$ des réponses).

Tableau 3: Répartition des réponses des enquêtés entre les pratiques liées au stockage des graines d'A. macrostachya

\begin{tabular}{|l|c|}
\hline \multicolumn{1}{|c|}{ Pratiques en matière de stockage de stockage } & Proportion des réponses en \% \\
\hline Graines stockées seules & 81,62 \\
\hline Graines stockées avec d'autres denrées (stockage mixte) & 18,38 \\
\hline \multicolumn{1}{|c|}{ Denrées associées aux graines d'A. macrostachya pour un stockage mixte } \\
\hline Niébé & 36 \\
\hline Arachide & 28 \\
\hline Soja & 12 \\
\hline Sorgho & 12 \\
\hline Gombo & 4 \\
\hline Mil & 4 \\
\hline Sésame & 4 \\
\hline
\end{tabular}

Contraintes biotiques et nature des pertes liées à la conservation des graines d'A. macrostachya

Agents causant les dégâts aux stocks : De l'avis des producteurs, trois agents causent des dégâts aux stocks d'A. macrostachya. Il s'agit des insectes, des rongeurs et des moisissures. Selon ces producteurs enquêtés
$(80,71 \%)$, les insectes constituent les principaux agents de dégradation des stocks. Viennent ensuite les rongeurs (rats et souris) et les moisissures avec respectivement 12,86 et $6,43 \%$ des répondants (Figure 4 ). 


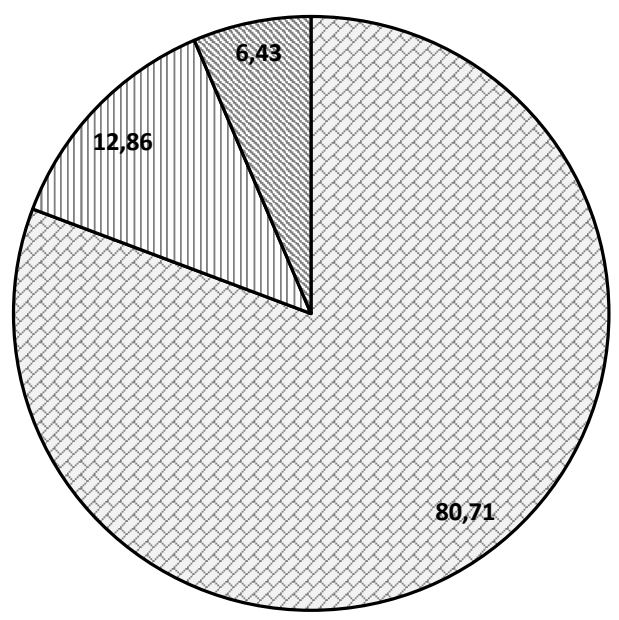

Figure 4 : Perceptions des enquêtés sur les principaux agents causant des dégâts aux stocks de $A$. macrostachya

Nature des dégâts: Plusieurs types de dégâts sont susceptibles d'être occasionnés par les différents déprédateurs (Tableau 4). Ainsi, selon les producteurs, on a comme dégâts et par ordre d'importance la perforation des graines $(66,81 \%$ des réponses), des graines brisées ou endommagées $(19,03 \%$ des réponses), la pourriture des graines et la perte de leur pouvoir germinatif avec respectivement $8,41 \%$ et $5,75 \%$ des réponses. Notons cependant que la perforation des graines et/ou gousses est perceptible déjà pendant la récolte ( $75 \%$ des enquêtés).

Tableau 4 : Perception des répondants sur la nature des dégâts dus aux déprédateurs des stocks

\begin{tabular}{|l|c|}
\hline Nature des dégâts & Proportion des réponses (\%) \\
\hline Graines perforées & 66,81 \\
\hline Graines brisées ou endommagées & 19,03 \\
\hline Pertes du pouvoir germinatif & 5,75 \\
\hline Pourriture des graines & 8,41 \\
\hline
\end{tabular}

Durée de conservation en bon état: Selon les producteurs, la durée pendant laquelle les graines d'A. macrostachya peuvent être conservées en bon état varie significativement en présence ou en absence de méthodes de lutte ( $W=15350, P<0,0001 ;$ Figure 5). La durée moyenne de conservation des graines d'A. macrostachya en bon état en présence de méthode de lutte est de 419 jours (environ 14 mois) tandis qu'elle est de 13 jours, soit moins d'un mois en absence de méthode de lutte. 


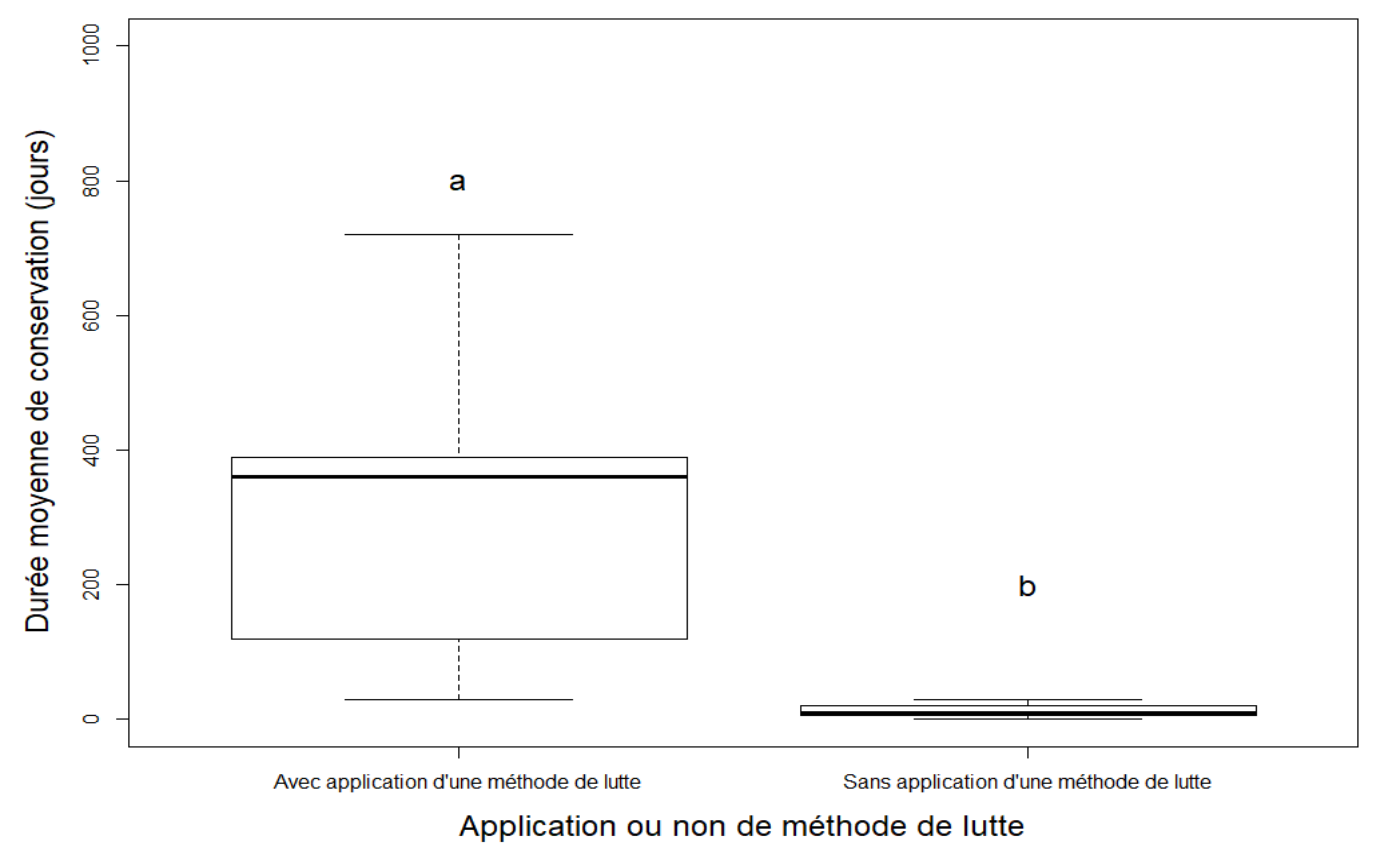

Figure 5 : Durée moyenne de conservation des graines d'A. macrostachya en présence et en absence de méthode de lutte

\section{Méthodes de protection}

Principales méthodes de protection utilisées : Quatre (4) méthodes de protection sont utilisées pour la conservation des graines d'A. macrostachya dans la zone d'enquête (Figure 6). II s'agit de la précuisson à la chaleur, du stockage hermétique, de l'utilisation d'un produit chimique et d'une substance minérale (la cendre). Ces méthodes n'ont cependant pas la même importance. La méthode la plus utilisée de l'avis des enquêtés, est la précuisson à la chaleur (62,96\% des réponses).

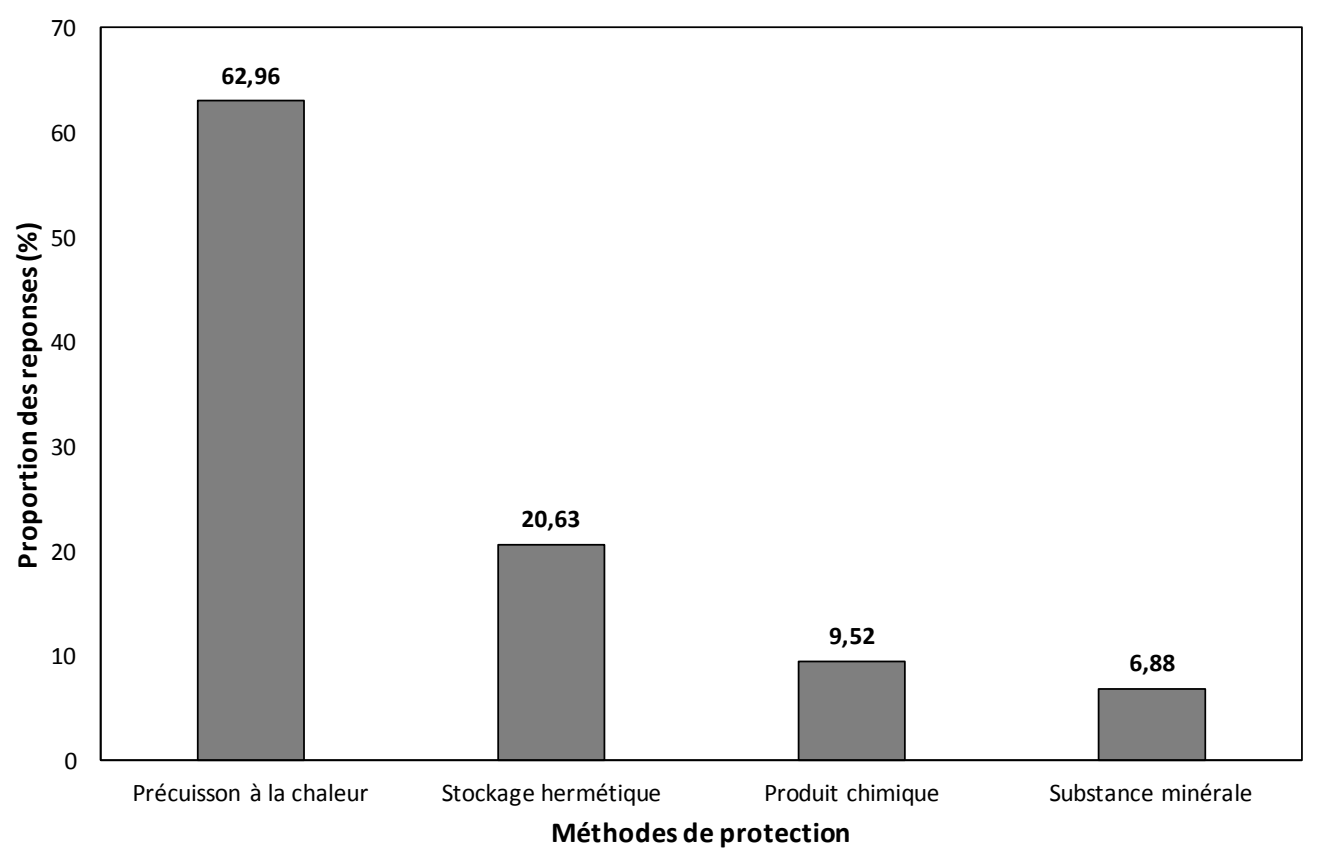

Figure 6 : Réponses des enquêtés sur les méthodes de protection des stocks d'A. macrostachya 
Description des méthodes de protection

Précuisson à la chaleur: il existe deux variantes. La première consiste à mettre les graines déjà séchées dans une marmite métallique contenant un peu d'eau et posée sur du feu doux. A l'aide d'une écumoire, on retourne continuellement les graines pour éviter qu'elles ne crament. L'opération dure une vingtaine de minutes. A la fin de l'opération on sèche à nouveau ces graines pendant une journée avant de les conserver. A la différence de cette première variante, le chauffage des graines dans la deuxième variante se fait à sec.

Produit chimique : il s'agit du phostoxin. C'est le nom commercial d'un fumigant dont la matière active est le phosphure d'hydrogène. Sous forme de comprimé, on l'emballe dans un tissu avant de l'introduire dans le stock. Stockage hermétique: il consiste à introduire les graines dans les bidons en plastique de vingt litres (20l) de contenance avant de les entreposer. Ces graines sont bien tassées dans les bidons de manière à éliminer le maximum d'air intergranulaire.

Substance minérale : On mélange les graines avec de la cendre avant de les conserver.
Destinée des stocks: Les objectifs visés par les exploitants en conservant les graines d'A. macrostachya sont variables (Tableau 5). Ainsi par ordre d'importance, $27,16 \%$ des enquêtés stockent les graines pour une utilisation pendant des cérémonies (mariages, fiançailles, funérailles, ...) tandis que d'autres ont pour objectifs la vente pour satisfaire certains besoins ponctuels $(25,56 \%)$, la consommation durant toute l'année $(23,64 \%)$ ou encore la vente quand les prix augmentent $(19,17 \%)$. Le prix moyen d'une assiettée yoruba augmente significativement du mois de Décembre au mois d'Août $(\mathrm{W}=875, \mathrm{P}<0,0001$; Figure 7). Ainsi, la moyenne des prix d'une assiettée yoruba (équivalent à environ $2,45 \mathrm{~kg}$ ) passe de 1260 FCFA pendant la période de récolte en décembre, à 1800 FCFA en août pendant la période de soudure. De l'avis des producteurs enquêtés, le marché local constitue le principal point d'écoulement des graines d'A. macrostachya avec $88 \%$ des réponses. Ouagadougou et les autres villes du Burkina sont par ordre d'importance les deux autres points d'écoulement.

Tableau 5: Réponses des enquêtés sur les usages qu'ils font des graines après stockage

\begin{tabular}{|l|c|}
\hline Objectifs visés & Proportion des réponses (\%) \\
\hline Utilisation pendant les cérémonies & 27,16 \\
\hline Vente pour satisfaire des besoins ponctuels (maladies, besoins d'argent etc.,) & 25,56 \\
\hline Consommation durant toute l'année & 23,64 \\
\hline Commercialisation quand les prix des ventes augmentent & 19,17 \\
\hline Consommation pendant la période de soudure & 4,47 \\
\hline
\end{tabular}

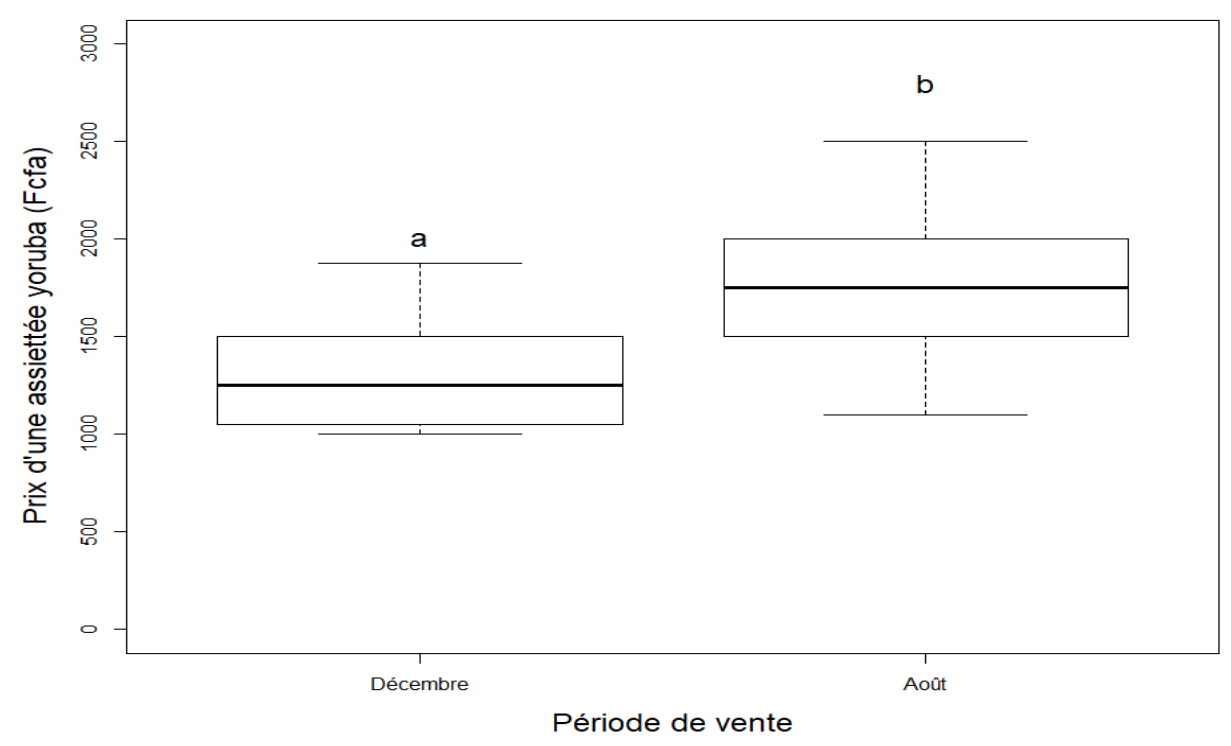

Figure 7 : Prix moyen d'une assiettée yoruba en fonction de la période de vente 


\section{DISCUSSION}

Cette étude nous a permis de mieux cerner les différentes pratiques des producteurs de zamné, en majorité des femmes $(87,10 \%$ des enquêtées) depuis la récolte jusqu'à la conservation et éventuellement la vente des graines de zamné. Dans la province du Boulkiemdé, l'exploitation et la conservation du zamné sont essentiellement réalisées par la gent féminine. Ce sont donc les femmes qui s'intéressent majoritairement à la filière zamné d'où cette représentativité parmi les enquêtés. Après la récolte, qui a lieu en décembre, la plupart des exploitants sèchent les gousses, les battent et les vannent puis sèchent les graines avant le stockage. Le séchage des gousses qui se fait en général à la maison et à l'air libre durant 1 et 7 jours vise la préservation de la valeur nutritionnelle et du pouvoir germinatif des graines (en réduisant leur respiration) et la protection de ces graines contre les bactéries et les champignons par la diminution de leur taux d'humidité (Scheepens et al., 2011). Le battage qui se fait manuellement à l'aide de bâton, permet de libérer les graines des gousses alors que le vannage est une opération destinée à débarrasser les graines de toute impureté y compris les éventuels insectes présents. Après ces différentes opérations, les graines sont stockées préférentiellement dans les sacs en polypropylène de $50 \mathrm{~kg}$. Des travaux similaires effectués au centre du Bénin par Chougourou et alavo (2011) ont montré que $59,17 \%$ des enquêtés utilisent les sacs en jute de 50 à $100 \mathrm{~kg}$ pour stocker les graines de légumineuses car selon eux le stockage en sac facilite la vérification des stocks et la manutention. Les bidons de 201 et les jarres en terre cuite sont aussi couramment utilisés pour le stockage des graines d'A. macrostachya. Ce sont des structures répandues qui permettent en effet le stockage de petites quantités de graines. Ces différentes structures permettent de stocker les graines seules, sans mélange avec d'autres denrées. Quelques enquêtés stockent les graines dans les greniers en banco car ces structures favorisent le stockage mixte c'est-àdire qu'elles permettent de stocker les graines d'A. macrostachya avec d'autres denrées notamment le niébé et l'arachide. L'inconvénient majeur de ce système de stockage mixte est qu'il pourrait favoriser le transfert de ravageurs d'une des denrées vers une autre. En effet selon une étude réalisée par Sankara (2012) Callosobruchus maculatus, ravageur des stocks de niébé, arrive à accomplir son cycle de développement avec plus ou moins de succès sur des substrats occasionnels que sont le voandzou et le pois d'angole et à s'y maintenir plusieurs années de suite. La prédominance d'une ou plusieurs de ces structures de stockage dans une région pourrait être liée aux moyens de lutte utilisés contre les déprédateurs et à la disponibilité des matériaux de construction au niveau local (Waongo et al., 2013). Les dégâts causés aux stocks sont surtout dus aux insectes et ils se matérialisent entre autres par la perforation des graines. Selon Monge et Huignard (2011), les pertes post récolte dues aux attaques des insectes ravageurs nuisibles peuvent être considérées sur le plan quantitatif par des pertes pondérales et sur le plan qualitatif par la diminution de la valeur nutritive et de la capacité germinative. Une enquête similaire réalisée par Sankara et al. (2016), a montré que la majorité des dégâts occasionnés aux stocks de niébé et de voandzou dans la région des Hauts Bassins au Burkina Faso, est due aux insectes ; il en est de même pour les stocks de céréales des producteurs de la zone sud soudanienne du Burkina Faso (Waongo et al., 2013). On note aussi une forte proportion de réponses relatives aux graines brisées probablement due à l'action des rongeurs et à la manutention. Selon Inge de Groot (2004), les rongeurs endommagent les denrées stockées en mangeant une partie du produit, en les souillant de leurs excréments et en véhiculant des maladies dangereuses pour l'Homme. La majorité des enquêtés affirme observer la perforation des gousses pendant la récolte ce qui suggère une infestation des graines avant la récolte et le stockage. Cette infestation primaire se poursuit pendant le stockage entrainant des dégâts aux stocks en moins d'un mois de conservation selon la majorité des enquêtés. Il està noter que les dégâts peuvent déjà être perceptibles en moins d'une semaine de stockage. L'infestation des graines d'Acacia au champ a également été signalée sur d'autres espèces du même genre, notamment sur Acacia raddiana au Sénégal (Delobel et al., 2003) et Acacia tortilis en Tunisie (Mejri et al., 2015). Pour faire face à ces déprédateurs, la méthode de protection des stocks d'A. macrostachya la plus utilisée est la précuisson à la chaleur. Selon les enquêtés, ce procédé permet non seulement de tuer les adultes déjà perceptibles à la récolte, mais également d'éliminer les œufs et larves qui y sont présents, et donc d'arrêter tout processus de développement larvaire en cours. Cette méthode s'apparente au traitement à l'eau chaude utilisée pour la conservation des légumineuses à graines et du voandzou en cosse dans le centre du Bénin (Chougourou et alavo, 2011). Le stockage hermétique, un produit chimique et une substance minérale sont aussi des méthodes utilisées pour la protection des stocks. Les substances minérales notamment la cendre remplit les vides entre les 
graines et constituent une barrière à la progression des femelles cherchant à pondre (Cissokho et al., 2015) alors que le stockage hermétique provoque la mort des insectes adultes ou des larves par anoxie (Huignard et al., 2011). Le produit chimique utilisé par $10 \%$ des répondants est le Phostoxin. C'est un fumigant non homologué par le Comité Sahélien des Pesticides (CSP, 2016) pour la conservation des denrées, ce qui suscite quand même quelques inquiétudes. En effet, selon Zongo et al. (2015), $77 \%$ des insecticides utilisés pour la conservation du niébé par les commerçants et producteurs dans la région centrale du Burkina Faso, ne sont ni reconnus, ni destinés à la conservation des aliments; ce qui provoque plusieurs troubles chez les consommateurs; les plus importantes étant les intoxications alimentaires pouvant conduire à la mort. Contrairement à la précuisson à la chaleur qui semble spécifique à cette denrée, ces trois dernières méthodes sont aussi utilisées pour la protection des stocks de niébé et de voandzou dans la région des Hauts-Bassins du Burkina Faso (Sankara et al., 2016). Ces différentes méthodes de protection permettent de stocker les graines

\section{CONCLUSION}

Cette étude nous a permis de faire, pour la première fois, un état des lieux sur la gestion post-récolte des graines d'A. macrostachya dans la province du Boulkiemdé au Burkina Faso. II ressort qu'après récolte et séchage complet, les gousses sont battues puis vannées avant d'être conservées dans des structures variées parmi lesquelles, les sacs en nylon sont les plus représentés.

\section{RÉFÉRENCES BIBLIOGRAPHIQUES}

Arbonnier M., 2000. Arbres, arbustes et lianes des zones sèches d'Afrique de l'ouest. CIRAD-MNHNUICN, 541p.

Chougourou D. C. et alavo T. B. C., 2011. Systèmes de stockage et méthodes endogènes de lutte contre les insectes ravageurs des légumineuses à grains entreposées au centre du Bénin. Revue CAMES-Série A, 12 (2), 137-141.

Cissokho P. S., Gueye M. T., Sow E. H. et Diarra K., 2015. Substances inertes et plantes à effet insecticide utilisées dans la lutte contre les insectes ravageurs des céréales et légumineuses au Sénégal et en Afrique de l'Ouest. Int. J. Biol. Chem. Sci. 9(3), 1644-1653

Comite Sahélien des Pesticides, 2016. Liste globale des pesticides autorisés version 2016. Secrétariat permanent du CSP INSAH, Bamako, 34p. en bon état durant une période moyenne de 14 mois. Cependant, Le stockage hermétique avec les bidons, la précuisson à la chaleur et l'utilisation de la cendre qui sont des méthodes de conservation traditionnelle sont difficilement extrapolables à des stocks plus importants (Huignard et al., 2011). La conservation de ces graines vise différents objectifs. Elles sont conservées pour être consommées après cuisson pendant les cérémonies de mariage (religieux et civil), baptême et fiançailles. De ce fait, la denrée est de plus en plus prisée tant en milieu rural qu'urbain. Le Zamnè est aussi utilisé comme mets quotidiens durant toute l'année avant les prochaines récoltes. Dans le but de se procurer beaucoup d'argent, certains producteurs font de la spéculation. Ainsi, ces graines sont vendues principalement sur les marchés locaux, quand les prix de vente augmentent surtout pendant la période de soudure en août où les prix augmentent de façon substantielle. Une bonne maitrise de la gestion post récolte du Zamnè pourrait permettre de faire de cette filière une alternative prometteuse de lutte contre la pauvreté.

Les insectes qui sont les plus redoutables déprédateurs infestent les graines avant la récolte et poursuivent leur développement dans les systèmes de stockage. La précuisson à la chaleur est la méthode de lutte la plus utilisée pour faire face aux insectes ravageurs. Les graines ainsi conservées ont de multiples usages dont la vente qui est une source de revenus non négligeable.

Dagnelie P., 1998. Statistiques théoriques et appliquées. Tome2 : Inférence statistique à une et à deux dimensions. Paris et Bruxelles, De Boeck et Larcier, $659 \mathrm{p}$.

Delobel A., Tran M. et Danthu P., 2003. Insectes consommateurs des graines d'Acacia raddiana en Afrique de l'Ouest : les bruches. In : Un arbre au désert, Acacia raddiana, IRD éditions, Paris, pp 285-299.

El-Miziani I., Lhaloui S., El Bouhssini M., Dahan R., Lamiri A. Gaboun F., \& Abbad Andaloussi F, 2016. Étude des dégâts qualitatifs et quantitatifs dus aux Bruches sur les légumineuses au Maroc. Revue Marocaine de Protection des Plantes N09, 83-99

Fandohan P., Hell K., Marasas W.F.O., 2003. Infection of mayse by Fusarium specifies and contamination 
with fumonisin in Africa. African Journal of Biotechnology 2, 510-579.

FAO. 2016. Légumineuses : des graines nutritives pour un avenir durable, 196p.

Fontès J. et Guinko S., 1995. Carte de la végétation et de l'occupation du sol au Burkina Faso, notice explicative. Toulouse Cedex France, 67p.

Gueye M. T., Seck. D., Wathelet J-P. et Lognay G., 2011. Lutte contre les ravageurs des stocks de céréales et de légumineuses au Sénégal et en Afrique occidentale : synthèse bibliographique. Biotechnol. Agron. Soc. Environ. 2011 15(1), 183-194.

Hagberg S., Gomgnimbou M. and Some' B., 1996. Forêts classées et terres des ancêtres au Burkina Faso. Working Paper No 3: Department of Cultural Anthropology, Uppsala University, 69 p.

Hama-Ba F., Siedogo M., Ouedraogo M., Dao A., Dicko H. M., and Diawara B., 2017. Modalités de consommation et valeur nutritionnelle des légumineuses alimentaires au Burkina Faso. Afr. J. Food Agric. Nutr. Dev. ; 17(4), 12871-12888

Huigard J., Glitho I. A., Monge J. P., Regnault-Roger C., 2011. Insectes ravageurs des graines de légumineuses : Biologie des Bruchidae et lutte raisonnée et Afrique. Editions Quae, 146p

Hunter D. and Fanzo J., 2013. Agricultural biodiversity, diverse diets and improving nutrition. In: Fanzo $\mathrm{J}$, Hunter D, Mikkelsen $\mathrm{J} \mathrm{H}$ and Langohr R. (eds), Diversifying food and diets: using agricultural biodiversity to improve nutrition and health. Issues in agricultural biodiversity (London), Earthscan, p. 1-13.

Inge de Groot, 2004. Protection des céréales et des légumineuses stockées. Agrodok 18,74p

Institut National de la Statistique et de la Démographie, 2006. Recensement général de la population, Tableau 1.1-Répartition de la population résidente par commune et selon le sexe, 41p.

Institut National de la Statistique et de la Démographie, 2016. Annuaire Statistique 2015, 397p.

Kristensen M. and Balslev H., 2003. Perceptions, use and availability of woody plant among the Gourounsi in Burkina Faso. Biodiversity and Conservation 12, 1715-1739.

Mejri M., Jamâa M. L. B., Grami M., 2015. Les bruches ravageurs de l'Acacia tortilis en Tunisie, infestation et méthodes de lutte. Editions universitaires européennes, $72 p$
Monge J-P. et Huignard J., 2011. Biologie des hyménoptères parasitoïdes des larves et des nymphes de Bruchinae. In : Insectes ravageurs des graines de légumineuses: Biologie des Bruchidae et lutte raisonnée et Afrique. Editions Quae, 65-81

Sankara F., 2012. Étude des conditions de transfert de Callosobruchus maculatus (Coleoptera: Chrysomelidae, Bruchidae) et de son parasitoïde Dinarmus basalis Rond. (Hymenoptera: Pteromalidae) sur des complexes d'hôtes secondaires. Thèse de doctorat unique, Université de Ouagadougou, $130 \mathrm{p}$.

Sankara F., Gondé Z., Sanou A. G. et Somda I., 2016. Diagnostic participatif des pratiques paysannes post-récolte et les contraintes de stockage de deux légumineuses cultivées dans la région des Hauts-Bassins du Burkina : cas du niébé, Vigna unguiculata (L.) Walp. et du voandzou, Vigna subterranea (L.) Verdc. International Journal of Innovation and Applied Studies, n03, vol. 16, 646-656.

Savadogo A., llboudo A. J and Traoré S., 2011. Nutritional potential of Acacia macrostachya (Reichend) ex DC seeds of Burkina Faso: Determination of Chemical Composition and Functional Properties. Journal of Applied Sciences Research 7 (7), 1057-1062.

Scheepens P., Hoevers R., Arulappan F. X. et Pesch G., 2011. Le stockage des produits agricoles : Serie Agrodok n031, 83p.

Schelin M., Mulualem T., Ingalill E., Sawadogo L. and Per Christer O., 2004. Predispersal seed predation in Acacia macrostachya, its impact on seed viability, and germination responses to scarification and dry heat treatments. New Forest 27, 251-267.

Sembène M. P., 1996. Les pertes post-récoltes, l'exemple de la bruche de l'arachide (Caryedon serratus Ol.) au Sénégal : Fonds Documentaire ORSTOM, 11p.

Thiombiano D. N. E., Nieyidouba L., Dibong D. S., Boussim I. J. et Belem B., 2012. Le rôle des espèces ligneuses dans la gestion de la soudure alimentaire au Burkina Faso. Sécheresse 23, 86-93.

Waongo A., Yamkoulga M., Dabiré-Binso C. L, Niango Ba M. et Sanon A., 2013. Conservation postrécolte des céréales en zone sud-soudanienne du Burkina Faso: Perception paysanne et 
évaluation des stocks. Int. J. Biol. Chem. Sci. (7) 3, 1157-1167.

Wittig R., Schmidt M. et Thiombiano A., 2004. Cartes de distribution des espèces du genre Acacia L. au Burkina Faso. Études flor. vég. Burkina Faso 8, 19-26.

Zida D., 2007. Impact of forest management regimes on ligneous regeneration in the sudanian savanna of Burkina Faso. Acta Universitatis Agriculturae Sueciae, doctoral Thesis $n^{0} 2007$ : 66, 22p.

Zongo S., llboudo Z., Waongo A., Gnankiné O., Doumma A., Sembène M. et Sanon A. 2015. Risques liés à l'utilisation des insecticides au cours du stockage du niébé (Vigna Unguiculata L. Walp.) dans la région centrale du Burkina Faso. Rév. CAMES, Vol.03 Num.01. 2015, 24-31. 